\title{
Conhecimento dos Profissionais de Saúde da Família Sobre Úlcera por Pressão
}

\author{
Knowledge of Family Health Professionals About Pressure Ulcer
El Conocimiento de los Profesionales de Salud de la Familia Acerca de las Úlceras por Presión

Antonio Dean Barbosa Marques', July Grassiely de Oliveira Branco², Rochelle da Costa Cavalcante Maria da Conceição Coelho Brito ${ }^{4}$, Samira Rêgo Martins de Deus ${ }^{5}$, Maria Helena Barros Araújo Luz 6

\begin{abstract}
RESUMO
Este estudo objetivou avaliar o conhecimento dos profissionais que atuam na Estratégia Saúde da Família sobre avaliação, classificação e prevenção da úlcera por pressão. Tratou-se de um estudo descritivo-exploratório, realizado em julho de 2014, no município de Crateús, no estado do Ceará, Brasil. Os dados foram coletados por meio de um questionário validado. Participaram 12 profissionais, sendo quatro médicos, quatro enfermeiros e quatro técnicos em Enfermagem. Os médicos atingiram a média de 75,6\%; os enfermeiros de 74,4\% e os técnicos de Enfermagem de 62,5\% dos acertos. Conclui-se que há falta de conhecimento com relação às diretrizes existentes sobre prevenção, classificação e tratamento da úlcera por pressão entre os profissionais que atuam na atenção básica. A educação permanente pode ser uma estratégia eficaz para aprimorar o conhecimento desses profissionais.
\end{abstract}

DESCRITORES: Úlcera por pressão. Saúde da família. Conhecimento. Estomaterapia.

ABSTRACT

This study aimed at assessing the knowledge of professionals that work in the Brazilian Family Health Strategy regarding the rating, classification and prevention of pressure ulcer. This was a descriptive and exploratory study, conducted in July 2014 in the city of Crateús, Ceará State, Brazil. Data were collected using a validated questionnaire. Twelve professionals took part in the survey, including four physicians, four nurses and four Nursing technicians. The physicians obtained an average of $75.6 \%$, while nurses reached $74.4 \%$ and Nursing technicians got $62.5 \%$ of correct answers. We concluded that there is still a lack of knowledge as to the existing guidelines of prevention, classification, and treatment of pressure ulcer among professionals working in the primary care. Continued education might be an effective strategy to improve the knowledge of these professionals.

DESCRIPTORS: Pressure ulcer. Family health. Knowledge. Stomatherapy.

\footnotetext{
'Enfermeiro. Mestrando em Saúde Coletiva pela Universidade de Fortaleza (UNIFOR). Professor no Ensino Médio Integrado (EMI) do Instituto CENTEC e Professor do Curso de Graduação em Enfermagem da Faculdade Princesa do Oeste (FPO) - Crateús (CE), Brasil. Endereço para correspondência: Antonio Dean Barbosa Marques - Rua Rui Monte, 131 - apto. 101 - Antonio Bezerra - CEP: 60360-640 - Fortaleza (CE), Brasil. - E-mail: antonio-dean@hotmail.com ²Enfermeira. Mestre em Saúde Coletiva pela UNIFOR - Fortaleza (CE), Brasil.

${ }^{3}$ Enfermeira. Mestranda em Saúde Coletiva pela UNIFOR. Professora EMI do Instituto CENTEC - Fortaleza (CE), Brasil.

${ }^{4}$ Enfermeira. Mestre em Saúde da Família pela Universidade Federal do Ceará (UFC). Professora da Universidade Estadual Vale do Acaraú (UVA) -

Sobral (CE), Brasil.

${ }^{5}$ Enfermeira. Doutoranda em Farmacologia pela UFC. Professora da Universidade Estadual do Piauí (UESPI) - Teresina (PI), Brasil.

${ }^{6}$ Enfermeira. Doutorado em Enfermagem pela Escola de Enfermagem Anna Nery da Universidade Federal do Rio de Janeiro (EEAN/UFRJ). Especialista em Estomaterapia pela Universidade de São Paulo (USP). Professora-Associada da UFPI - Teresina (PI), Brasil.

Artigo recebido em: 19/11/2015 - Aceito para publicação em: 23/12/2015
} 


\section{RESUMEN}

Este estudio tuvo como objetivo evaluar lo conocimiento de los profesionales que trabajan en la Estrategia de Salud Familiar sobre evaluación, clasificación y prevención de la úlcera por presión. Se trató de un estudio descriptivo y exploratorio realizado en julio de 2014 en la ciudad de Crateús, en Ceará, Brasil. Los datos fueron recolectados a través de un cuestionario validado. El equipo se compuso por 12 profesionales, incluyendo cuatro médicos, cuatro enfermeros/ as y cuatro técnicos/as de Enfermería. Los médicos atingieron una media del 75,6\%, los enfermeros obtuvieron 74,4\% y $62,5 \%$ de los técnicos de Enfermería de los aciertos. Se concluye que hay una falta de conocimientos sobre los protocolos existentes de prevención, clasificación y tratamiento de las úlceras por presión entre los profesionales que trabajan en atención primaria. La educación continua puede ser una estrategia eficaz para mejorar el conocimiento de esos profesionales.

DESCRIPTORES: Úlcera por presión. Salud de la familia. Conocimiento. Estomaterapia.

\section{INTRODUÇÃO}

A ocorrência de úlcera por pressão (UP) tem sido considerada um grave problema de saúde pública que atinge todas as camadas da população na atualidade. No passado, os idosos eram os mais acometidos; entretanto, em decorrência do aumento no número de acidentes automobilísticos, passou-se a observar esse fenômeno entre jovens e adultos em idade produtiva ${ }^{1}$.

A National Pressure Ulcer Advisory Panel (NPUAP) e a European Pressure Ulcer Advisory Panel (EPUAP) definem UP como as áreas localizadas de lesões necróticas situadas na pele ou em tecidos subjacentes, as quais tendem a se desenvolver geralmente sobre uma proeminência óssea, decorrente do resultado de pressão, forças de cisalhamento e fricção ou da combinação desses três fatores ${ }^{2}$.

O desenvolvimento da UP é um fenômeno complexo, que envolve vários fatores associados, sendo que a imobilidade por tempo prolongado é o mais importante fator de risco para pacientes acamados e cadeirantes. É importante lembrar que a UP pode também ocorrer em períodos considerados curtos. Dependendo do paciente, o intervalo de duas horas, ou até menos, pode ser tempo suficiente para o desenvolvimento da lesão ${ }^{3}$.

Em virtude de seu caráter iatrogênico, o surgimento da UP pode ser evitável. Por esse motivo, as UP são consideradas indicadores de qualidade técnico-científica, tanto no âmbito da atenção primária como na atenção hospitalar especializada ${ }^{1}$. Para o monitoramento e os danos na assistência à saúde, o Ministério da Saúde (MS) lançou a Portaria $n^{\circ} 529$ de $1^{\circ}$ de abril de 2013, a qual institui o Programa Nacional de Segurança do Paciente e tem por objetivo geral contribuir para a qualificação do cuidado em todos os estabelecimentos de saúde do território nacional ${ }^{4}$.
Nesse contexto, os enfermeiros vêm atuando de maneira persistente sobre essa problemática, tornando-se um tema em evidência para a sua prática clínica ${ }^{5}$.

Em função da transição epidemiológica, impulsionada pelos avanços na área da saúde, as pessoas estão vivendo mais. Há muitos indivíduos acamados ou com algum tipo de mobilidade prolongada assistida por equipes de saúde da familia (ESF), que, em grande parte, constitui-se de idosos, os quais necessitam de cuidados contínuos por parte da família, dos cuidadores e de profissionais da área da saúde.

O paciente acamado ou com restrição de mobilidade no domicílio está suscetível a apresentar problemas complexos e, muitas vezes, tem seu estado de saúde agravado com a presença de UP, em que podem vivenciar dor, sofrimento, comprometimento sistêmico, quase sempre associado à infecção, às secreções com odor fétido e à alteração da imagem corporal, podendo ocorrer baixa autoestima, desmotivação e insegurança. As UP causam grande impacto também na vida dos familiares e cuidadores, ocasionando muita sobrecarga emocional e física. Os enfermeiros não podem se limitar a serem meros observadores ${ }^{5}$. Além disso, nota-se que o custo financeiro se torna elevado no tratamento desses pacientes e, quando mal conduzido ou tratado inadequadamente, pode resultar em sérias complicações e no aumento da mortalidade ${ }^{6}$.

Durante o acompanhamento do paciente acamado em domicílio, o enfermeiro da Estratégia Saúde da Família deve realizar as orientações necessárias para o cuidado preventivo de lesões; acompanhar com avaliações periódicas os acamados com risco para UP; manter registros de suas ações e capacitar os profissionais de nível médio de Enfermagem, cuidadores e familiares, em função do frequente contato 
com o paciente, ajudando-os nas orientações e nos cuidados preventivos ${ }^{6}$.

O interesse pela temática surgiu a partir da atuação como enfermeiro da Estratégia Saúde da Família. Observou-se uma dificuldade por parte de alguns profissionais que atuavam na Estratégia Saúde da Família, como médicos, enfermeiros, técnicos de Enfermagem e agentes comunitários de saúde, em abordar um paciente com UP. Em geral, cada profissional orientava os cuidadores de pacientes acamados de maneira diferente, enquanto os profissionais de Enfermagem apresentavam homogeneidade em relação aos principais cuidados.

As UP representam uma das principais complicações que acometem pacientes acamados, por isso, na busca da qualidade de assistência, destaca-se a necessidade de conhecimento científico dos profissionais de saúde relacionado à UP.

Baseando-se nesse contexto, surgiram as seguintes questões norteadoras: Qual o conhecimento de profissionais que atuam na Estratégia Saúde da Família sobre a prevenção, a avaliação e o tratamento de UP? Existe associação entre os escores e o conhecimento de membros da Estratégia Saúde da Família e as variáveis sociodemográficas e/ou formação profissional?

Espera-se que este estudo possa ampliar o conhecimento na área de Estomaterapia, a fim de subsidiar novas reflexões dos profissionais de saúde, em especial os que atuam na saúde da família, no sentido de proporem ou definirem novas estratégias de assistência dentro do âmbito da atenção básica à saúde.

O estudo teve como objetivo analisar o conhecimento dos profissionais que atuam na Estratégia Saúde da Família sobre a avaliação, a classificação e a prevenção da UP.

\section{METODOLOGIA}

Tratou-se de um estudo de natureza descritiva-exploratória, com abordagem quantitativa, realizado no município de Crateús, no estado do Ceará. $\mathrm{O}$ município conta com um total de 16 ESF cadastradas no MS; dessas, quatro foram sorteadas aleatoriamente para a aplicação do teste de conhecimento.

Para inclusão das ESF, estas deveriam ter uma equipe mínima composta por médico, enfermeiro e técnico de Enfermagem. Os profissionais deveriam estar regularmente inscritos no Cadastro Nacional de Estabelecimento de Saúde (CNES), ter no mínimo um ano de atuação profissional em saúde da família, para os enfermeiros e técnicos em Enfermagem, e no mínimo um mês para o médico. Foram excluídos sujeitos que não atenderam a esses critérios, assim como os que não aceitaram participar.

A coleta de dados foi realizada em julho de 2014, por meio de um instrumento composto por itens referentes aos dados sociodemográficos e ao teste de conhecimento de Pieper e $\mathrm{Mott}^{7}$, que foi validado e adaptado em estudo anterior realizado no Brasil. O conteúdo do material é proveniente das recomendações de diretrizes norte-americanas para a prevenção da UP e é constituído por 41 afirmações verdadeiras ou falsas, com oito itens sobre avaliação e classificação da UP e 33 sobre prevenção da UP. Foram suprimidos três itens por não se adequarem ao contexto da atenção básica, restando sete sobre avaliação e classificação, e 31 sobre prevenção da UP, perfazendo 38 itens.

Para cada uma das afirmações, o participante deveria selecionar uma resposta, considerando as opções: verdadeiro (V), falso (F) e não sei (NS) - modelo Likert. Para cada acerto, atribuiu-se um ponto. Os acertos corresponderam às afirmações verdadeiras respondidas como $\mathrm{V}$ ou falsas, como F. Para as respostas erradas ou para aquelas respondidas como NS, o escore atribuído foi zero. O escore total do teste de conhecimento correspondeu à soma de todas as respostas corretas.

Posteriormente, os dados coletados foram digitados em planilha Excel, utilizando-se a técnica de dupla digitação, para análise no programa Statistical Package for Social Science, versão 21.0 (SPSS). Para a análise, foram considerados os escores dos três grupos de profissionais e não os isolados para cada sujeito.

As associações foram verificadas pelo teste de $\mathrm{F}$ (ANOVA), utilizado para realizar a comparação da pontuação média do teste de conhecimento de Pieper e Mott entre os três tipos de profissionais; pelo teste $t$ de Student, que foi utilizado para realizar a comparação da pontuação média do teste de conhecimento com a variável sexo e estado civil; e pelo teste de significância da coeficiência de correlação, emprego para verificar a significância estatística da correlação das variáveis tempo de profissão e tempo de trabalho com a pontuação no teste de conhecimento.

O estudo foi aprovado pelo Comitê de Ética em Pesquisa da Universidade Estadual Vale do Acaraú (UVA), conforme estabelece o Conselho Nacional de Saúde (CNS), por meio da Resolução 466/12, que se refere às pesquisas 
envolvendo seres humanos, com o parecer 674.823 e CAEE 30685714.5.0000.5053.

\section{RESULTADOS}

Participaram da pesquisa 12 profissionais da ESF, sendo quatro médicos, quatro enfermeiros e quatro técnicos de
Enfermagem. A distribuição dos participantes, segundo as suas características sociodemográficas, está apresentada na Tabela 1 .

Quanto à idade, a média dos profissionais ficou em torno de 38,75 anos. Os médicos com a média de 43,25 anos, enfermeiros com 38,5 anos e técnicos em Enfermagem com 34,5 anos. A respeito do gênero, observou-se uma distribuição igual para todos os profissionais, 6 (50\%) eram

Tabela 1. Distribuição dos participantes da pesquisa, segundo suas características sociodemográficas. Crateús, 2014.

\begin{tabular}{|c|c|c|c|c|c|c|}
\hline \multirow[t]{2}{*}{$\begin{array}{l}\text { Características } \\
\text { sociodemográficas }\end{array}$} & \multicolumn{2}{|c|}{$\begin{array}{l}\text { Médico } \\
(n=4)\end{array}$} & \multicolumn{2}{|c|}{$\begin{array}{l}\text { Enfermeiro } \\
\qquad(n=4)\end{array}$} & \multicolumn{2}{|c|}{$\begin{array}{l}\text { Técnico em } \\
\text { Enfermagem } \\
(n=4)\end{array}$} \\
\hline & $\mathrm{n}$ & $\%$ & $\mathrm{n}$ & $\%$ & $n$ & $\%$ \\
\hline \multicolumn{7}{|l|}{ Idade (anos) } \\
\hline$<30$ & 1 & 25,0 & 0 & 0 & 0 & 0 \\
\hline 30 a 40 & 1 & 25,0 & 2 & 50,0 & 4 & 100,0 \\
\hline 40 a 50 & 1 & 25,0 & 2 & 50,0 & 0 & 0 \\
\hline$\leq 60$ & 1 & 25,0 & 0 & 0 & 0 & 0 \\
\hline Total & 4 & 100,0 & 4 & 100,0 & 4 & 100,0 \\
\hline \multicolumn{7}{|l|}{ Gênero } \\
\hline Feminino & 1 & 25,0 & 2 & 50,0 & 3 & 75,0 \\
\hline Masculino & 3 & 75,0 & 2 & 50,0 & 1 & 25,0 \\
\hline Total & 4 & 100,0 & 4 & 100,0 & 4 & 100,0 \\
\hline \multicolumn{7}{|l|}{ Estado civil } \\
\hline Solteiro (a) & 2 & 50,0 & 1 & 25,0 & 1 & 25,0 \\
\hline Casado (a) & 2 & 50,0 & 3 & 75,0 & 2 & 50,0 \\
\hline Divorciado (a) & 0 & 0 & 0 & 0 & 1 & 25,0 \\
\hline Total & 4 & 100,0 & 4 & 100,0 & 4 & 100,0 \\
\hline \multicolumn{7}{|c|}{ Tempo de formação profissional (anos) } \\
\hline$<5$ & 1 & 25,0 & 0 & 0 & 0 & 0 \\
\hline 05 a 10 & 1 & 25,0 & 1 & 25,0 & 1 & 25,0 \\
\hline 10 a 15 & 0 & 0 & 3 & 75,0 & 2 & 50,0 \\
\hline 15 a 20 & 0 & 0 & 0 & 0 & 1 & 25,0 \\
\hline 20 a 25 & 1 & 25,0 & 0 & 0 & 0 & 0 \\
\hline$\geq 25$ & 1 & 25,0 & 0 & 0 & 0 & 0 \\
\hline Total & 4 & 100,0 & 4 & 100,0 & 4 & 100,0 \\
\hline \multicolumn{7}{|c|}{ Tempo de atuação profissional na Estratégia Saúde da Família (anos) } \\
\hline$<05$ & 1 & 25,0 & 0 & 0 & 2 & 50,0 \\
\hline 05 a 10 & 2 & 50,0 & 1 & 25,0 & 2 & 50,0 \\
\hline 10 a 15 & 1 & 25,0 & 3 & 75,0 & 0 & 0 \\
\hline Total & 4 & 100,0 & 4 & 100,0 & 4 & 100,0 \\
\hline \multicolumn{7}{|l|}{ Formação } \\
\hline Especialização & 3 & 75,0 & 4 & 100,0 & & \\
\hline Mestrado & 1 & 25,0 & 0 & 0 & & \\
\hline Doutorado & 0 & 0 & 0 & 0 & & \\
\hline Total & 4 & 100,0 & 4 & 100,0 & & \\
\hline
\end{tabular}


homens e 6 (50\%) eram mulheres. Já, quando analisado por categoria profissional, surgiram algumas diferenças. Três (75\%) dos médicos eram homens; os enfermeiros apresentaram a mesma distribuição para os sexos masculino e feminino (50\%); em relação aos técnicos em Enfermagem, houve predominância do gênero feminino, que correspondeu a 3 (75\%). Em relação ao estado civil desses profissionais, $2(50 \%)$ dos médicos eram solteiros e a outra metade era casada; para os enfermeiros, 3 (75\%) eram casados e apenas $1(25 \%)$ era solteiro; já para os técnicos, $1(25 \%)$ era solteiro, 2 (50\%) casados e 1 (25\%) declarou ser divorciado.

Em relação ao tempo de formação profissional, obteve-se uma média de 13,7 anos, variando conforme a categoria profissional. Um profissional médico (25\%) estava apenas com um mês de formado, os demais, três (75\%), obtiveram a média de 17 anos. Os enfermeiros ficaram com a média de 11,5 anos de formação e técnicos com 12,5 anos. Quanto ao tempo de atuação profissional na Estratégia Saúde da Familia, a média desses sujeitos ficou em torno de 6,75 anos. Ao contrastar esse tempo com a categoria, os médicos apresentaram seis anos completos, nove anos para os enfermeiros e 5,25 anos para os técnicos em Enfermagem. Sobre a titulação dos profissionais de nível superior, três (75\%) dos médicos possuem especialização, quatro (100\%) dos enfermeiros possuem especialização e um médico (25\%) possui mestrado.

A Tabela 2 apresenta os resultados obtidos pelos profissionais nas áreas de avaliação e classificação da UP, do teste de conhecimento.

Considerando os resultados integrais do teste, os médicos atingiram a média de $75,6 \%$; os enfermeiros, $74,4 \%$ e os técnicos de Enfermagem, 62,5\% dos acertos.

Observou-se que, quando analisados individualmente, nenhum dos participantes conseguiu atingir a média de acertos igual ou superior a $90 \%$, preconizada pelo teste. As notas máximas foram de $81,6 \%$ para um participante médico e um enfermeiro e $76,3 \%$ para um técnico em

Tabela 2. Percentual de acertos dos participantes da pesquisa no teste de conhecimento de Pieper e Mott, segundo os itens sobre avaliação e classificação da úlcera por pressão. Crateús, 2014.

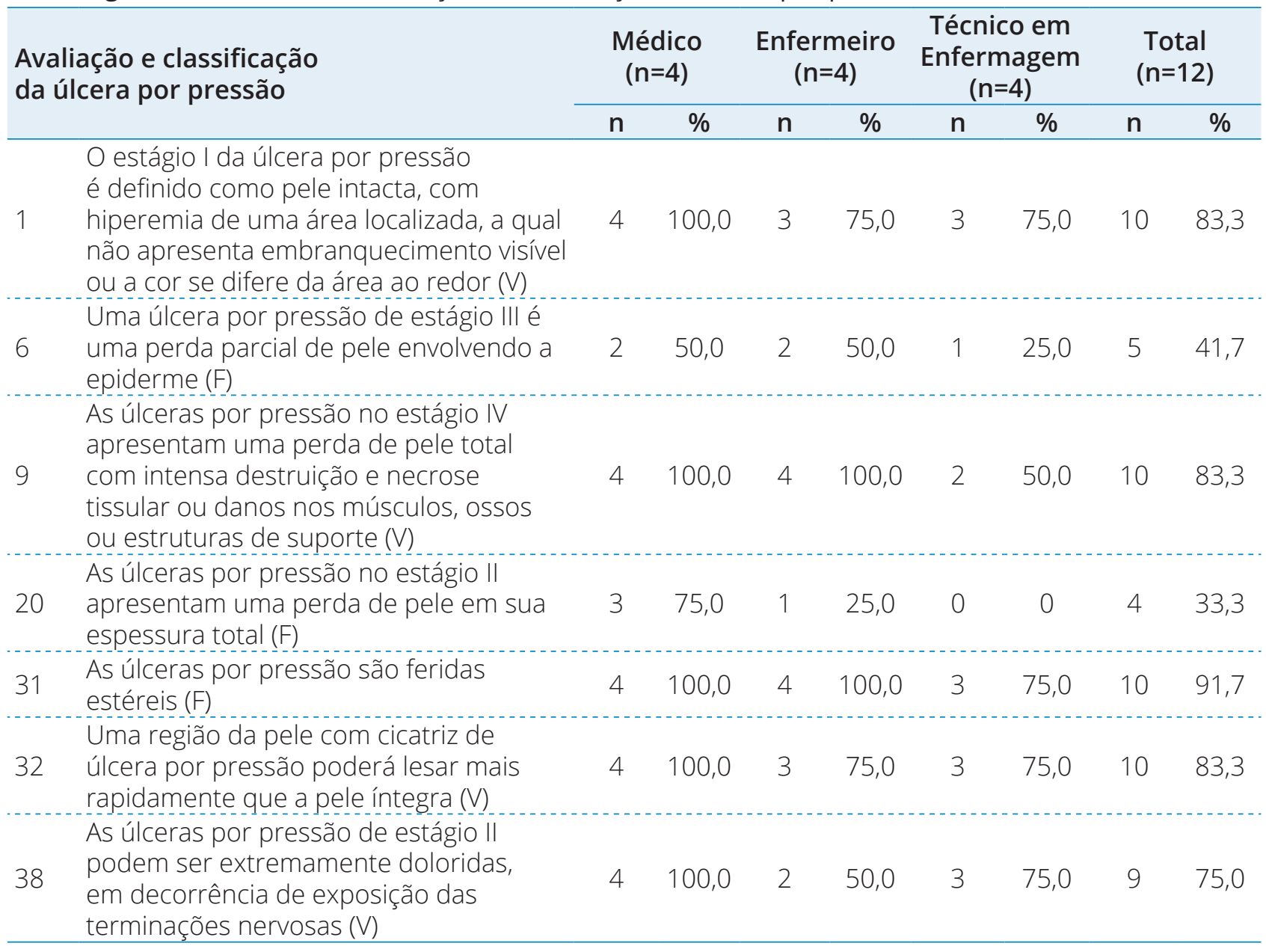

V: verdadeiro; F: Falso. 
Enfermagem. As menores notas registradas foram 68,4\% para um médico, 60,6\% para um enfermeiro e $47,4 \%$ para um técnico em Enfermagem.

Destaca-se que, nos itens referentes à avaliação e à classificação da UP, apenas no item 31 os participantes obtiveram o resultado superior do estabelecido pelo teste, nos demais itens, o resultado foi abaixo. Nos itens 1, 9, 31 e 32, os participantes obtiveram $83,3 \%$ dos acertos; no item, a assertiva foi de 75\%; item 6, 41,6\%; no item número 20, os profissionais obtiveram o menor resultado $(33,3 \%)$. Os médicos conseguiram obter $100 \%$ de acertos em quatro itens $(9,31$, 32 e 38) e os enfermeiros em 2 (9 e 31).

A Tabela 3 apresenta os resultados obtidos pelos profissionais nas áreas de prevenção de UP, do teste de conhecimento.

Nos itens referentes à prevenção da UP, composta ao todo por 31, em 14 itens os participantes tiveram mais do que $90 \%$ dos acertos, sendo que em 7 itens, número 23, 26, $27,29,35,39$ e 40, todos os participantes obtiveram 100\% dos acertos; em 7 itens entre 70 e 89,9\% de acertos; em 2 itens entre 50 e 69\%; e em 8 apresentaram porcentagem de acertos menor que $50 \%$.
Os aspectos com menor índice de acertos pelos profissionais foram referentes à inspeção sistemática e posição em decúbito lateral com 41,7\% de acertos, ao posicionamento quanto à elevação da cabeceira do leito e movimentação de paciente restrito ao leito com 25\%, massagem em proeminências ósseas e uso de luvas d'água ou ar com 16,7\% e reposicionamento de pacientes restritos ao leito e almofadas tipos d'água ou ar com a média de 8,3 de acertos.

Os profissionais médicos não pontuaram em cinco itens, 3, 11, 13, 14 e 17, e pontuaram sozinhos em 2 itens, 3 e 16; os enfermeiros não pontuaram apenas em 1 item, 16, mas foram os únicos a pontuarem em 2 itens, 11 e 14, e os técnicos em Enfermagem não pontuaram em cinco itens, 5, $11,14,15$ e 16 .

Por meio do teste de F (ANOVA), verificou-se a existência de algum grupo com média de pontos diferente dos demais. Com valor $\mathrm{p}=0,183$, foi rejeitada a hipótese de que exista algum grupo diferente dos demais. $\mathrm{O}$ teste $t$ de Student para comparação de grupos independentes foi comparado à soma de pontos para outras variáveis. Não se obteve diferença para as variáveis sexo $(p=0,560)$ e estado civil $(p=0,746)$. Já o

Tabela 3. Percentual de acertos dos participantes da pesquisa no teste de conhecimento de Pieper e Mott, segundo os itens sobre prevenção da úlcera por pressão. Crateús, 2014.

\begin{tabular}{|c|c|c|c|c|c|c|c|c|c|}
\hline \multirow{2}{*}{\multicolumn{2}{|c|}{$\begin{array}{l}\text { Prevenção da úlcera } \\
\text { por pressão }\end{array}$}} & \multicolumn{2}{|c|}{$\begin{array}{l}\text { Médico } \\
(n=4)\end{array}$} & \multicolumn{2}{|c|}{$\begin{array}{l}\text { Enfermeiro } \\
\qquad(\mathrm{n}=4)\end{array}$} & \multicolumn{2}{|c|}{$\begin{array}{l}\text { Técnico em } \\
\text { Enfermagem } \\
(n=4)\end{array}$} & \multicolumn{2}{|c|}{$\begin{array}{l}\text { Total } \\
(n=12)\end{array}$} \\
\hline & & $n$ & $\%$ & $\mathrm{n}$ & $\%$ & $\mathrm{n}$ & $\%$ & $\mathrm{n}$ & $\%$ \\
\hline 2 & $\begin{array}{l}\text { Os fatores de risco para o } \\
\text { desenvolvimento da úlcera } \\
\text { por pressão são: imobilidade, } \\
\text { incontinência, nutrição inadequada e } \\
\text { alteração do nível de consciência (V) }\end{array}$ & 4 & 100,0 & 3 & 75,0 & 2 & 50,0 & 9 & 75,0 \\
\hline 3 & $\begin{array}{l}\text { Todos os pacientes em risco para } \\
\text { úlcera por pressão devem ter uma } \\
\text { inspeção sistemática da pele pelo } \\
\text { menos uma vez por semana (F) }\end{array}$ & 0 & 0 & 2 & 50,0 & 3 & 75,0 & 5 & 41,7 \\
\hline 4 & $\begin{array}{l}\text { O uso de água quente e sabonete } \\
\text { podem ressecar a pele e aumentar } \\
\text { o risco para úlcera por pressão }(\mathrm{V})\end{array}$ & 2 & 50,0 & 3 & 75,0 & 2 & 50,0 & 7 & 58,3 \\
\hline 5 & $\begin{array}{l}\text { É importante massagear as regiões } \\
\text { das proeminências ósseas, se } \\
\text { estiverem hiperemiadas }(F)\end{array}$ & 1 & 25,0 & 1 & 25,0 & 0 & 0 & 2 & 16,7 \\
\hline 8 & $\begin{array}{l}\text { Os cremes, curativos transparentes } \\
\text { e curativos de hidrocoloides do tipo } \\
\text { extrafino auxiliam na proteção da } \\
\text { pele contra os efeitos da fricção (V) }\end{array}$ & 4 & 100,0 & 4 & 100,0 & 3 & 75,0 & 11 & 91,7 \\
\hline 10 & $\begin{array}{l}\text { Uma ingestão dietética adequada de } \\
\text { proteínas e calorias deve ser mantida } \\
\text { durante a doença/hospitalização }(\mathrm{V})\end{array}$ & 4 & 100,0 & 4 & 100,0 & 3 & 75,0 & 11 & 91,7 \\
\hline
\end{tabular}


Tabela 3. Continuação.

\begin{tabular}{|c|c|c|c|c|c|c|c|c|c|}
\hline \multirow{2}{*}{\multicolumn{2}{|c|}{$\begin{array}{l}\text { Prevenção da úlcera } \\
\text { por pressão }\end{array}$}} & \multicolumn{2}{|c|}{$\begin{array}{l}\text { Médico } \\
(n=4)\end{array}$} & \multicolumn{2}{|c|}{$\begin{array}{l}\text { Enfermeiro } \\
\qquad(n=4)\end{array}$} & \multicolumn{2}{|c|}{$\begin{array}{l}\text { Técnico em } \\
\text { Enfermagem } \\
(n=4)\end{array}$} & \multicolumn{2}{|c|}{$\begin{array}{l}\text { Total } \\
(n=12)\end{array}$} \\
\hline & & $\mathrm{n}$ & $\%$ & $\mathrm{n}$ & $\%$ & $\mathrm{n}$ & $\%$ & $\mathrm{n}$ & $\%$ \\
\hline 11 & $\begin{array}{l}\text { Os pacientes que ficam restritos ao } \\
\text { leito devem ser reposicionados a } \\
\text { cada três horas (F) }\end{array}$ & 0 & 0 & 1 & 25,0 & 0 & 0 & 1 & 8,3 \\
\hline 12 & $\begin{array}{l}\text { Uma escala com horários para } \\
\text { mudança de decúbito deve ser } \\
\text { utilizada para cada paciente com } \\
\text { presença ou em risco para úlcera } \\
\text { por pressão }(\mathrm{V})\end{array}$ & 3 & 75,0 & 4 & 100,0 & 4 & 100,0 & 11 & 91,7 \\
\hline 13 & $\begin{array}{l}\text { As luvas d'água ou de ar aliviam a } \\
\text { pressão nos calcâneos (F) }\end{array}$ & 0 & 0 & 1 & 25,0 & 1 & 25,0 & 2 & 16,7 \\
\hline 14 & $\begin{array}{l}\text { As almofadas tipo rodas d'água ou } \\
\text { ar auxiliam na prevenção da úlcera } \\
\text { por pressão (F) }\end{array}$ & 0 & 0 & 1 & 25,0 & 0 & 0 & 1 & 8,3 \\
\hline 15 & $\begin{array}{l}\text { Na posição em decúbito lateral, o } \\
\text { paciente com presença de úlcera } \\
\text { por pressão ou em risco deve ficar } \\
\text { em um ângulo de } 30^{\circ} \text { em relação ao } \\
\text { colchão do leito (V) }\end{array}$ & 3 & 75,0 & 2 & 50,0 & 0 & 0 & 5 & 41,7 \\
\hline 16 & $\begin{array}{l}\text { No paciente com presença de úlcera } \\
\text { por pressão ou em risco, a cabeceira } \\
\text { da cama não deve ser elevada em } \\
\text { um ângulo maior do que } 30^{\circ} \text { se não } \\
\text { houver contraindicação médica (V) }\end{array}$ & 3 & 75,0 & 0 & 0 & 0 & 0 & 3 & 25,0 \\
\hline 17 & $\begin{array}{l}\text { O paciente que não se movimenta } \\
\text { sozinho deve ser reposicionado a } \\
\text { cada dua horas enquanto sentado }\end{array}$ & 0 & 0 & 2 & 50,0 & 1 & 25,0 & 3 & 25,0 \\
\hline
\end{tabular}
na cadeira $(F)$

O paciente com mobilidade limitada e que pode mudar a posição do

18 corpo sem ajuda deve ser orientado a realizar o alívio da pressão a $4 \quad 100,0 \quad 3 \quad 75,0 \quad 3 \quad 75,0 \quad 10$ 83,3 cada 15 minutos, enquanto estiver sentado na cadeira (V) O paciente com mobilidade limitada e que pode permanecer na cadeira

19 deve ter uma almofada no assento para proteção da região das proeminências ósseas (V) A pele do paciente em risco para

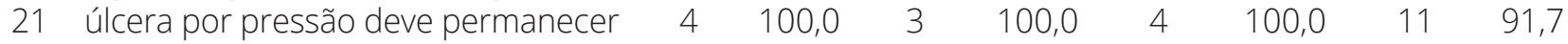
limpa e livre de umidade $(V)$

As medidas para prevenir novas

22 lesões não precisam ser adotadas continuamente quando o paciente

$\begin{array}{llllllll}3 & 75,0 & 3 & 75,0 & 3 & 75,0 & 9 & 75,0\end{array}$
já possui úlcera por pressão (F) Lençóis móveis ou forros devem

23 ser utilizados para transferir ou movimentar pacientes que não se movimentam sozinhos (V) 
Tabela 3. Continuação.

\section{Prevenção da úlcera por pressão}

A mobilização e a transferência de pacientes que não se movimentam

24 sozinhos devem ser sempre realizadas por duas ou mais pessoas (V) No paciente com condição crônica que não se movimenta sozinho,

25 a reabilitação deve ser iniciada e incluir orientações sobre prevenção e tratamento da úlcera por pressão (V)

Todo paciente que não deambula

26 deve ser submetido à avaliação de risco para o desenvolvimento de úlcera por pressão (V)

Os pacientes e familiares devem ser orientados quanto às causas

27 e aos fatores de risco para o desenvolvimento de úlcera por pressão $(V)$

As regiões de proeminências ósseas

28 podem ficar em contato direto uma com a outra (F)

Todo paciente em risco para

29 desenvolver úlcera por pressão deve ser colocado em superfície (colchão) redutora de pressão (V) A pele, quando macerada pela

30 umidade, danifica-se mais facilmente (V)

Uma boa maneira de diminuir a

34 pressão nos calcâneos é mantê-los elevados do leito (V)

Todo o cuidado administrado

35 para prevenir ou tratar as úlceras por pressão não precisa ser documentado (F)

Cisalhamento é a força que ocorre

36 quando a pele adere a uma superfície e o corpo desliza (V)

37

A fricção pode ocorrer ao movimentarse o paciente sobre o leito $(V)$

$4 \quad 100,0$ 4

100,0

4

100,0

12

100,0
Total $(n=12)$

$\begin{array}{llllllll}\mathrm{n} & \% & \mathrm{n} & \% & \mathrm{n} & \% & \mathrm{n} & \%\end{array}$

$\begin{array}{llllllll}3 & 75,0 & 4 & 100,0 & 4 & 100,0 & 11 & 91,7\end{array}$

$\begin{array}{llllllll}4 & 100,0 & 4 & 100,0 & 4 & 100,0 & 12 & 100,0\end{array}$

$\begin{array}{llllllll}3 & 75,0 & 4 & 100,0 & 3 & 75,0 & 10 & 83,3\end{array}$

83,3

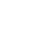


teste de significância do coeficiência de correlação, quando cruzadas as variáveis de tempo de formação e/ou atuação na Estratégia Saúde da Família com o número de acertos de cada profissional, indicou não haver correlação estatisticamente significativa.

\section{DISCUSSÃO}

No que concerne o resultado integral do teste, os médicos atingiram a média de 75,6\%; os enfermeiros, $74,4 \%$; os técnicos de Enfermagem, $62,5 \%$ dos acertos. Notou-se que, quando analisados individualmente, nenhum dos participantes conseguiu obter a média de acertos igual ou superior a $90 \%$ preconizada pelo teste. As notas máximas foram de $81,6 \%$ para um participante médico e um enfermeiro e $76,3 \%$ para um técnico em Enfermagem. As menores notas registradas foram: $68,4 \%$ para um médico, $60,6 \%$ para um enfermeiro e 47,4\% para um técnico em Enfermagem.

Grande parte das ESF possui pessoas acamadas ou com imobilidade prolongada, muitas são idosos, necessitando de uma assistência planejada com o intuito de prevenir complicações. Nesse sentido, o MS instituiu a Portaria no 2.029, de 24 de agosto de 2011, que dispõe sobre a atenção domiciliar no âmbito do Sistema Único de Saúde, definindo-a como um conjunto de atividades prestadas no domicílio a pessoas clinicamente estáveis, que exijam intensidade de cuidados acima das modalidades ambulatoriais, mas que possam ser mantidas em casa, por equipe exclusiva, composta minimamente por médico, enfermeiro e técnico de Enfermagem, além de equipe e serviço de apoio ${ }^{8}$.

Nesse sentido, o enfermeiro emerge com a responsabilidade sobre a pessoa acamada ou com imobilidade prolongada, tendo em vista que este profissional coordena, implementa, supervisiona as ações da equipe, alimenta os sistemas de informação, realiza treinamentos e capacitações do cuidado domiciliar, contribuindo para a eficácia e eficiência do serviço de saúde ${ }^{6}$. Estes sujeitos são os profissionais que diagnosticam e prescrevem as intervenções capazes de prevenir as UP, por meio do contato direto com o cliente, ou indireto, na integração do cuidador informal na prestação de cuidados. Portanto, é de suma importância refletir sobre todos os aspectos a eles inerentes, de forma a atribuir aos enfermeiros formação técnico-científica adequada para ultrapassar esta problemática 5 .
Os resultados do teste de conhecimento de Pieper e Mott, considerando o total de acertos, mostraram que tanto o conhecimento dos médicos $(75,6 \%)$, como dos enfermeiros $(74,4 \%)$ e técnicos de Enfermagem (62,5\%) foram insatisfatórios. Mesmo analisados individualmente, nenhum profissional conseguiu atingir o nível considerado adequado, que é preconizado em $90 \%$ ou mais dos itens do teste.

Os dados revelam que ambos os profissionais de saúde da família apresentam déficits de conhecimento em algumas áreas referentes ao tema, entretanto os técnicos de Enfermagem são os que possuem menos conhecimento.

No Canadá, Sinclair et al. ${ }^{9}$ implementaram e avaliaram uma série de workshops para dois níveis de profissionais de Enfermagem, antes e em dois momentos após o evento, utilizando o teste de conhecimento de Pieper e Mott ${ }^{7}$. Concluíram que os escores dos enfermeiros foram superiores aos dos técnicos em Enfermagem, em todos os momentos.

Pancorbo-Hidaldo et al. ${ }^{10}$, em estudo realizado com a equipe de Enfermagem de hospitais, unidades de saúde e centro de atendimento ao idoso na Espanha, investigaram o nível do conhecimento destes profissionais sobre as diretrizes existentes de UP, e a utilização das mesmas na prática clínica. Tais achados evidenciaram que os fatores educacionais exercem grande influência no conhecimento, reverberando assim na prática cotidiana. O nível do conhecimento de intervenções preventivas foi 79,1\% e o de implementação na prática clínica, 68,1\%. Os enfermeiros que receberam instrução específica obtiveram os melhores resultados, com 77,7\% dos escores.

Nos Estados Unidos, Zulkowski, Ayello e Wexler ${ }^{11}$ avaliaram o conhecimento sobre a UP com o teste de Pieper e Mott (1995) em enfermeiros especializados em cuidados de feridas, certificação em outra especialidade e sem certificação. Os enfermeiros com especialização na área acertaram 89\%, os especialistas em outras áreas, $78 \%$ e os sem especialidades, $76 \%$.

Os estudos evidenciam que, apesar do avanço técnico-científico na área da saúde e de diretrizes que apontam recomendações para a prevenção e o tratamento da UP, dificuldades sobre essa temática ainda perduram e persistem em ordem mundial. Não foram identificados estudos, tanto internacionais como brasileiros, que avaliem o conhecimento de profissionais da atenção básica relacionados à questão do conhecimento para a prevenção da UP. Destacam-se vários estudos que avaliam o saber dos profissionais de saúde em âmbito hospitalar.

Em geral, os estudos que abordam a temática UP na atenção primária à saúde objetivam investigar as características 
sociodemográficas e clínicas e o risco para desenvolvimento de UP, os cuidados dispensados aos pacientes, a prevalência e as medidas de prevenção, os saberes e as práticas de cuidadores, além dos fatores que influenciam os cuidados na assistência domiciliária ${ }^{5,6,12-15}$.

No estudo realizado em um hospital universitário de nível terciário do interior paulista com 366 participantes, sendo 64,8\% auxiliares/técnicos de Enfermagem e 35,2\% enfermeiros, os resultados apontaram que os enfermeiros acertaram 79,4\% e os técnicos de Enfermagem, 73,6\% do teste de conhecimento. Por meio da identificação das áreas deficientes, pode-se nortear o planejamento de estratégias disseminadoras e que contribuam para a adoção de medidas preventivas pela equipe ${ }^{16}$.

O mesmo teste foi aplicado em um Hospital Universitário Sul Fluminense com auxiliares e técnicos de Enfermagem, enfermeiros, fisioterapeutas e médicos. Os participantes também não conseguiram atingir a média do teste. Os auxiliares e técnicos de Enfermagem acertaram, em média, 69,4\% dos itens do teste; os enfermeiros, 73,6\%; os fisioterapeutas, 79,2\%; e os médicos, $72,7 \%{ }^{17}$.

Um estudo realizado em Fortaleza buscou avaliar o risco para UP em idosos no domicílio, após o período de internação hospitalar, mediante aplicação da escala de Braden. Foi apontado que a avaliação de risco para UP em idosos no domicílio, após alta hospitalar, mostrou-se uma importante estratégia para a promoção da saúde desse público. Enfatiza-se a necessidade de orientações voltadas à prevenção de UP no domicílio, prestadas no momento da alta hospitalar ${ }^{14}$.

Um estudo foi realizado no $\mathrm{Brasil}^{3}$, buscando avaliar o impacto de uma intervenção educativa, utilizando o teste de conhecimento de Pieper e Mott ${ }^{7}$ adaptado. Identificou-se que, na fase de pré-intervenção, os enfermeiros obtiveram 86,4\% de acertos; entretanto, nenhum profissional participou da avaliação posterior. Os auxiliares e técnicos de Enfermagem obtiveram 74,3\% de acertos, na fase pré-intervenção, e 81,2\%, na pós-intervenção, realizada 20 semanas após o curso. A intervenção para o grupo dos técnicos de Enfermagem se mostrou significativa, possibilitando a melhoria nos resultados do teste.

Uma investigação conduzida em um hospital público no Piauí recentemente, que objetivou verificar o efeito das modalidades de ensino presencial e à distância no conhecimento de enfermeiros sobre UP, obteve diferenças entre as modalidades.
A média de acertos no teste para os participantes da modalidade de ensino presencial foi de $82,9 \%$ e para os participantes do ensino à distância, 88,3\%. A diferença de médias foi estatisticamente significante. $\mathrm{O}$ efeito da modalidade de ensino à distância no conhecimento dos enfermeiros sobre UP foi maior do que na modalidade de ensino presencial ${ }^{18}$.

\section{CONCLUSÃO}

A porcentagem média de acertos no teste de conhecimento de Pieper e Mott para os médicos foi de 75,6\%; para os enfermeiros $74,4 \%$ e para os técnicos de Enfermagem, $62,5 \%$. Mesmo quando analisados individualmente, nenhum dos participantes conseguiu atingir a média de acertos preconizada pelo teste de igual ou superior a $90 \%$. A maior nota obtida foi de $81,6 \%$ para um participante médico e um enfermeiro e $76,3 \%$ para um técnico em Enfermagem. As menores notas registradas foram: 68,4\% para um médico, $60,6 \%$ para um enfermeiro e $47,4 \%$ para um técnico em Enfermagem.

A partir dos resultados obtidos neste estudo, foi possível inferir que há deficiência de conhecimento sobre as diretrizes existentes a respeito de prevenção, classificação e tratamento da UP entre os profissionais que atuam na atenção básica no município de Crateús, no estado do Ceará.

Espera-se com esta pesquisa contribuir para o aperfeiçoamento e reflexão dos profissionais da área da saúde, especialmente o enfermeiro, que lida diretamente com o cuidado e tem a responsabilidade de buscar atualizações e aplicar medidas preventivas, reduzindo assim os casos de UP, como também dos gestores para que possam desenvolver políticas públicas para atender com qualidade a essa clientela, oferecendo condições de trabalho à equipe multiprofissional que atende o usuário em domicílio.

Fica evidenciada a necessidade de mais estudos, os quais avaliem o conhecimento dos profissionais. A educação permanente pode ser uma estratégia eficaz para aprimorar o conhecimento desses profissionais e repercutir diretamente na qualidade da assistência prestada, atendendo às necessidades de saúde da população.

Ressalta-se como limitação deste estudo a restrição do tamanho da amostra, cujos resultados são pontuais e não podem ser generalizados. 


\section{REFERÊNCIAS}

1. Carvalho ES. Como cuidar de pessoas com feridas: desafios para a prática multiprofissional. Salvador: Atualiza; 2012. p. 177-96.

2. National Pressure Ulcer Advisory Panel. European Pressure Ulcer Advisory Panel and Pan Pacific Pressure Injury Alliance. Prevention and Treatment of Pressure Ulcers: Quick reference guide. Cambridge Media: Osborne Park, Australia; 2014.

3. Fernandes LM, Caliri MH, Haas VJ. Efeito de intervenções educativas no conhecimento dos profissionais de enfermagem sobre prevenção de úlceras por pressão. Acta Paul Enferm. 2008;21(2):305-11.

4. Brasil. Portaria n. 529, de $1^{\circ}$ de abril de 2013. Institui o Programa Nacional de Segurança do Paciente (PNSP) [online]. Brasília (DF): Ministério da Saúde; 2013 [acesso 2014 Jun 18]. Disponível em: http://bvsms.saude.gov.br/bvs/ saudelegis/gm/2013/prt0529_01_04_2013.html

5. Rodrigues AM, Soriano JV. Fatores influenciadores dos cuidados de enfermagem domiciliários na prevenção de úlceras por pressão. Rev Enferm Referência. 2011;3(5):55-63.

6. Bezerra SM. Prevalência de úlcera por pressão e cuidados dispensados em acamados no domicilio. [Dissertação de mestrado]. Teresina: Universidade Federal do Piauí; 2010.

7. Pieper B, Mott M. Nurses' knowledge of pressure ulcer prevention, stating, and description. Adv Wound Care. 1995;8(3):34-48.

8. Brasil. Ministério da Saúde. Secretaria de Atenção à Saúde. Institui a Atenção Domiciliar no âmbito do Sistema Único de Saúde. Brasília: Ministério da Saúde; 2009.

9. Sinclair L, Berwiczonek H, Thurston N, Butler S, Bulloch G, Ellery $C$, et al. Evaluation of an evidence-based education program for pressure ulcer prevention. J Wound Ostomy Continence Nurs. 2004;31(1):43-50.
10. Pancorbo-Hidalgo PL, García-Fernández FP, LópezMedina IS, López-Ortega J. Pressure ulcer care in Spain: nurses' knowledge and clinical practice. J Adv Nurs. 2007;58(4):327-38.

11. Zulkowski K, Ayello EA, Wexler S. Certification and education: do they affect pressure ulcer knowledge in nursing? Adv Skin Wound Care. 2007;20(1):34-8.

12. Chayamiti EM, Caliri MH. Úlcera por pressão em pacientes sob assistência domiciliária. Acta Paul Enferm. 2010;23(1):29-34.

13. Pessoa EF, Carvalho JG, Bezerra SM. Prevalência de úlcera por pressão em pacientes acamados, cadastrados na Estratégia de Saúde da Família: um estudo de enfermagem. Rev Interdisciplinar NOVAFAPI. 2011;4(1):14-8.

14. Moraes GL, Araújo TM, Caetano JA, Lopes MO, Silva MJ. Avaliação de risco para úlcera por pressão em idosos acamados no domicílio. Acta Paul Enferm. 2012;25(spe1):7-12.

15. Ramos DO, Oliveira OS, Santos IV, Carvalho ES, Passos SS, Góis JA. Conhecimento de familiares acerca das úlceras por pressão e de seus direitos à reparação. Rev. Baiana de Enferm. 2014;28(1):23-30.

16. Miyazaki MY, Caliri MH, Santos CB. Conhecimento dos profissionais de enfermagem sobre prevenção da úlcera por pressão. Rev Latino-Am Enferm. 2010;18(6):1-10.

17. Fernandes NC, Amaral JP. Conhecimento da equipe multidisciplinar sobre prevenção, avaliação e tratamento de úlcera de pressão no hospital universitário sul Fluminense/ RJ. Estação Cient. 2012;1(spe):1-10.

18. Cavalcanti PA, Andrade EM. Efeito das modalidades de ensino presencial e a distância no conhecimento de enfermeiros sobre úlcera por pressão. Rev Estima. 2013;11(2):42. 